\title{
Atletismo e infância: a habilidade de correr em situação de teste e festival
}

\author{
Athletics and childhood: the skill to running in test and festival situation
}

\author{
Rute Estanislava Tolocka ${ }^{1, *}$ \\ Vitor AntonioCerignoni Coelho ${ }^{2}$ \\ Lucas Vinicius Marin Pedro ${ }^{3}$ \\ Samuel Bento da Silva ${ }^{4}$
}

\begin{abstract}
Resumo
Objetivo: O objetivo deste estudo foi determinar se o contexto onde a corrida é realizada afeta o nível da habilidade motora de correr. Métodos: O desempenho de 34 crianças, de ambos os sexos, de sete a nove anos de idade, foi analisado em contexto de teste e festival de atletismo, com os mesmos critérios. Resultados: A maioria das crianças, $80 \%$ no contexto de teste e $74 \%$ no festival, não atingiu o máximo desempenho. Os resultados foram significativamente melhores no contexto de festival, para todos os segmentos do corpo. Conclusões:Sugere-se que a motivação é chave para o desempenho motor e para isso, é necessário que a avaliação seja feita em contexto ecológico. Atividades recreativas de atletismo poderiam ser introduzidas nas aulas de Atividade Física como uma ferramenta para melhorar o desempenho da corrida.
\end{abstract}

Palavras-chave: atletismo, Educação Física, habilidade motora, corrida, criança.

\begin{abstract}
Objective: The aim of this study was to determine whether the context in which the race is held affects the level of motor ability to run. Methods: The performance of 34 children, both sexes, from seven to nine years old, was analyzed in test and athletics festival context, with the same criteria. Results: Most of the children, $80 \%$ in the test context and $74 \%$ in the festival, did not reach the maximum performance. The results were significantly better in the festival context, to all body segments. Conclusion: It is suggested that motivation is a key to motor performance and it is necessary that the assessment might be done in ecological context. Recreational athletics activities could be introduced in the Physical Activity classes as a tool to improve running performance.
\end{abstract}

Key-words: track and field, Physical Education, motor skills, running, child.
Afiliação dos autores

${ }^{1}$ Universidade Metodista de Piracicaba, Piracicaba, São Paulo, Brasil.

${ }^{2}$ Universidade Federal do Tocantins, Palmas, Tocantis, Brasil.

Palmas, Tocantis, Brasil.
${ }^{3}$ Prefeitura Municipal de Piracicaba, Piracicaba, São Paulo, Brasil. ${ }^{4}$ Prefeitura Municipal de Cosmópolis, São Paulo, Brasil.

*Autor correspondente

Curso de Ciências do Movimento Humano, Rodovia do Açúcar, km 156, CEP:13.400-911, Piracicaba, SP, Brasil.

e-mail:

rnupem@yahoo.uk

Conflito de interesses

Os autores declararam não haver conflito de interesses.

Processo de arbitragem

Recebido: 21/06/2017 Aprovado: 20/04/2018 


\section{Introdução}

O atletismo é um dos esportes mais antigos da humanidade e envolve a prática de habilidades consideradas fundamentais para as crianças tanto em situações cotidianas, esportivas, lúdicas e ou recreativas ${ }^{1}$. A Educação Física tem debatido a presença do atletismo dentro da escola e as possibilidades de vivência, exploração e aquisição de habilidades motoras básicas ${ }^{2,3}$.

A prática desta modalidade pode estimular e contribuir com movimentos fundamentais para o cotidiano, mas para isto é necessário avaliar o nível de habilidade da criança a fim de estabelecer estratégias de intervenção e modificações da prática em aulas de Educação Física, para que as crianças potencializem suas habilidades.

A avaliação do nível de habilidades motoras básicas tem sido feita através de parâmetros nos quais se compara o movimento realizado pela criança com os critérios-referências sugeridos para uma determinada ação motora, em uma dada situação de testagem. Embora a literatura tenha proposto diferentes critérios de comparação, existe consenso em se observar o movimento por segmentos corporais e em classificar cada segmento em pelo menos três níveis de habilidade ${ }^{4}$.

Tais critérios são utilizados para avaliar a habilidade de correr, que é uma das ações mais importantes do atletismo e serve de base para execução de outras habilidades motoras, esportivas ou não. Esta habilidade tem sido objeto de estudo há bastante tempo ${ }^{5-9}$. Outros estudos concentraram-se em encontrar diferenças entre esta habilidade e questões de gênero, bem como em correlaciona-la com outras variáveis, tais como medidas antropométricas ou capacidades físicas ${ }^{10-13}$.

Foram realizados também estudos que verificaram o correr e variáveis ligadas a prática esportiva. Entre os estudos que observaram a modalidade de Atletismo, na última década (20072017), está a pesquisa de Katićet al. ${ }^{14}$ que verificou a relação entre nove componentes que mediam capacidades físicas básicas no contexto de teste e os resultados nas seguintes provas do atletismo: corrida de velocidade, lançamento de uma bola de $200 \mathrm{~g}$ e corrida de longa distância adaptada para crianças de sete anos e concluiu que as habilidades motoras básicas são influenciadas pelas capacidades físicas de força, resistência, velocidade e flexibilidade. $O$ estudo apontou que o desenvolvimento motor geral $e$ as habilidades motoras específicas do atletismo estão intimamente relacionados e ambos dependem dos aspectos biológicos e ambientais.

Panseraet al. ${ }^{15}$, Brauner e Valentini ${ }^{16}$ e Silva et al. ${ }^{17}$ estudaram outra habilidade básica associada ao atletismo, os autores verificaram que na habilidade de arremessar por cima do ombro as crianças apresentaram déficits em todos os componente da ação, o que não era mais esperado para a faixa etária analisada. Na capacidade de salto horizontal Panseraet al. ${ }^{15} \mathrm{e}$ Brauner e Valentini ${ }^{16}$ também verificaram que as crianças se encontravam abaixo do esperado, em vários componentes antes, durante e após os saltos. Contrapondo todas essas pesquisas Krebs et al. ${ }^{18}$ mostraram em um estudo realizado com cinquenta crianças, que a maioria delas se mostrava em níveis satisfatórios ou acima da média para as habilidades de corrida, arremesso e salto horizontal.

A partir desses achados, uma variável que parece interferir na execução do gesto habilidoso é o contexto onde o mesmo é executado, como apontado por Marques e Catenassi ${ }^{13}$, porém estudos que analisam a execução do movimento para além da situação de teste ainda são escassos.

Assim, o objetivo do estudo foi verificar se o contexto da corrida interfere no nível da habilidade motora de correr. Especificamente buscou-se avaliar o nível da habilidade de correr em situação de teste e de festival de atletismo e comparar o desempenho motor desta habilidade em ambos os contextos.

\section{Métodos}

ETrata-se de um estudo de campo, do qual participaram 34 crianças, de ambos os sexos, de sete a nove anos de idade, cursando a segunda série do ensino fundamental, de uma escola pública de um município do interior de São Paulo. Estas crianças faziam parte de um projeto para inclusão do atletismo na referida escola.

O nível de desenvolvimento da habilidade de correr foi analisado em dois contextos, sendo em ambos requisitados que as crianças corressem, o mais rápido possível, uma distância de 60 metros. Cada atividade foi efetuada em três tentativas intercaladas, sendo que cada criança realizava a atividade e esperava as outras crianças realizarem a prova para então fazer a próxima tentativa. A melhor das três execuções de cada criança foi utilizada como referência. A primeira situação foi de teste, conforme protocolo proposto por Mc Clenaghan e Gallahue $^{8}$, atualizado por Gallahue e Ozmun ${ }^{19}$ e utilizado em outros estudos com crianças brasileiras, como visto anteriormente.

O segundo contexto foi realizado em um festival de atletismo, na fase preliminar, onde as crianças realizaram as provas individualmente, o mais rápido possível. Foram seguidos os mesmos procedimentos que foram utilizados na realização dos testes para a gravação dos movimentos e respectivas análises.

A avaliação do estágio de execução de cada habilidade foi realizada através da observação das imagens, que após serem gravadas em Vídeo Tape, foram convertidas para linguagem digital, através do softwareStudio 11, que permitiu a visualização de todos os quadros para comparação com o check-list proposto por estes autores. Tal check-list verifica a posição das articulações de membros superiores, inferiores e tronco durante as tarefas e classifica cada um destes segmentos em inicial, elementar e maduro.

O desempenho global foi verificado a partir da atribuição de escores a cada nível de habilidade, sendo o estágio inicial $=1$, estágio elementar $=2$, estágio maduro $=3$ pontos; o escore final foi calculado a partir da soma dos escores em cada segmento analisado.

Foram utilizados testes para verificar a distribuição normal dos dados coletados, correlação de Sperman para relacionar o nível de habilidade dos diferentes segmentos corporais e o teste Wilcoxon para grupos relacionados a fim de comparar o resultado dos dois contextos. A distribuição dos dados foi ilustrada a partir da confecção de tabelas eboxplot.

Este estudo encontra-se de acordo as resoluções 196/96 e 251/97 do Conselho Nacional de Saúde e foi aprovado pelo Conselho de Ética em Pesquisa de uma Universidade, com o parecer no. 82/04. As crianças, bem como seus responsáveis, assinaram o termo de consentimento livre e esclarecido e a escola concedeu autorização para a realização do mesmo.

\section{Resultados}

Na situação de teste 27 crianças (80\%) demonstraram nível de habilidade de correr abaixo do esperado e apenas sete crianças $(20 \%)$ estavam no nível maduro, o mesmo aconteceu na situação de festival, com 25 crianças $(73 \%)$ e nove crianças $(27 \%)$ respectivamente.

Enquanto na situação de teste apenas $26 \%$ das crianças obtiveram score8 ou 9 , na situação de festival $94 \%$ das crianças atingiram estes scores, como pode ser visto na tabela 1 , que também apresenta a distribuição dos níveis de habilidade observados nos diferentes segmentos corporais e o desempenho final obtido em cada uma das situações.

Tabela 1

Distribuição do nível de habilidade de correr no contexto de teste e de festival de atletismo.

\begin{tabular}{|c|c|c|c|c|c|c|}
\hline No. 34 crianças & \multicolumn{3}{|c|}{ Situação de Teste } & \multicolumn{3}{|c|}{ Situação de Festival } \\
\hline Nível de habilidade & MS & $T$ & $\mathrm{MI}$ & MS & $T$ & MI \\
\hline Inicial & $21 \%$ & -- & -- & -- & -- & -- \\
\hline Elementar & $59 \%$ & $74 \%$ & $68 \%$ & $74 \%$ & $6 \%$ & $3 \%$ \\
\hline Maduro & $20 \%$ & $26 \%$ & $32 \%$ & $26 \%$ & $94 \%$ & $97 \%$ \\
\hline Score Total & \multicolumn{3}{|c|}{$\begin{array}{c}\text { Score Situação de } \\
\text { Teste }\end{array}$} & \multicolumn{3}{|c|}{$\begin{array}{c}\text { Score Situação de } \\
\text { Festival }\end{array}$} \\
\hline Score 5 & \multicolumn{3}{|c|}{$21 \%$} & & & \\
\hline Score 6 & \multirow{2}{*}{\multicolumn{3}{|c|}{$6 \%$}} & \multirow{2}{*}{\multicolumn{3}{|c|}{$3 \%$}} \\
\hline Score 7 & & & & \multirow{2}{*}{\multicolumn{3}{|c|}{$\begin{array}{c}3 \% \\
67 \%\end{array}$}} \\
\hline Score 8 & \multirow{2}{*}{\multicolumn{3}{|c|}{$\begin{array}{r}6 \% \\
20 \%\end{array}$}} & & & \\
\hline Score 9 & & & & \multicolumn{3}{|c|}{$27 \%^{*}$} \\
\hline
\end{tabular}

A Figura 1 ilustra o valor mínimo e máximo, a mediana e a distribuição dos dados no primeiro, segundo e terceiro quartis referentes ao desempenho das crianças e permite visualizar a diferença encontrada no desempenho das crianças nos dois contextos em que realizaram a corrida. Verifica-se que 0 desempenho do grupo nos dois contextos foi diferente em todas 
estas medidas, havendo concentração do desempenho no escore oito, onde a criança está madura em pelo menos dois, dos três segmentos analisados.

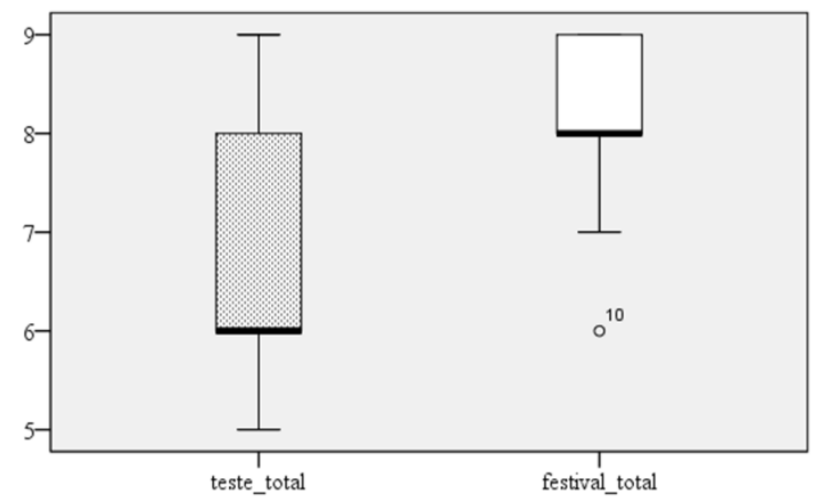

Figura 1. Nível de habilidade de correr

A diferença entre os dois contextos e o melhor desempenho na situação de festival foi confirmada pelo teste de Wilcoxon para dados não paramétricos, sendo significativamente diferente para os membros superiores $(p<0,05)$; para o tronco $(p<0,001)$; para os membros inferiores $(p<0,001)$; e para o score total $(p<0,001)$. Assim, houve diferença significativa do desempenho das crianças nos dois contextos representando um efeito maior para o contexto de festival, o desempenho da habilidade de correr em situação de festival foi melhor do que o obtido no teste.

\section{Discussão}

Os resultados mostraram que algumas crianças ficaram abaixo do esperado para a idade, esses resultados corroboram com o de Katicet al. ${ }^{14}$, Pansera et al. ${ }^{15}$, Brauner e Valentini ${ }^{16}$, Silva et al. ${ }^{17}$, Lorson e Goodway ${ }^{20}$ pois em todos esses estudos as crianças apresentaram o nível motor abaixo do esperado para a idade, já que o esperado é que as crianças apresentassem níveis maduros em todos os segmentos corporais a partir de sete anos de idade.

Essa defasagem no nível de habilidade motora pode ser ocasionada pela falta de estímulos proporcionados às crianças e há necessidade de propiciar mais vivências para que as crianças possam atingir níveis mais avançados de desenvolvimento e utilizarem estas habilidades de maneira mais efetiva em atividades da vida diária ou de prática esportiva, se assim o desejarem.

O segmento que se apresentou menos desenvolvido em ambas as situações foi 0 membro superior. Observa-se, entretanto, que na situação de teste, um número expressivo de crianças $(21 \%)$ apresentou estágio inicial de desenvolvimento no movimento de membros superiores, mas nenhuma delas o fez na situação de festival e que a porcentagem de crianças que havia demonstrado nível maduro de execução do movimento de tronco subiu de $26 \%$ na situação de teste para $94 \%$ no festival, o que demonstra que muitas crianças tinham o potencial para executar a habilidade com mais desenvoltura do que o fizeram na situação de teste, corroborando com Marques e Catanessi ${ }^{13}$ ao argumentarem que o contexto natural da execução do movimento favorece o desempenho da habilidade.

Assim, comparando-se os dois contextos, o de festival de atletismo se mostrou mais eficaz em todos os requisitos corporais (membros superiores, membros inferiores e troncos). Isto pode ter ocorrido, porque, segundo Blascovi-Assis ${ }^{21}$, a criança em situação de jogo demonstra melhor potencial de ação em determinadas habilidades, pois o contexto proporciona maiores estímulos às crianças, como torcida, manifestações emocionais e atitudes competitivas.

De acordo com Schimitd e Wrisberg ${ }^{22}$ esses elementos podem motivar a criança a executar as habilidades de forma mais eficiente. Rose Junior ${ }^{23}$ reforça que a motivação é algo que permite a criança estabelecer metas significativas de maneira que as atinja com excelência se superando constantemente.

A competição é considerada "motivo de realização", ou seja, é a melhoria ou manutenção da própria capacidade na atividade em que se é possível medir o desempenho e, embora o contexto competitivo possa levar ao êxito ou fracasso, a competição aparece também como um dos seis fatores que compõe o lúdico ${ }^{24}$ e está ligada à motivação para a realização da tarefa ${ }^{22}$.

Outra explicação é que embora o termo competição possa ser associado com perigo e disputa com o outro, ele não significa necessariamente conflito para com o outro, pois pode ser considerado como desafio individual, onde o desejo de se superar leva ao empenho para uma performance mais acurada $^{25}$

Assim, como a situação de teste não foi realizada de forma lúdica ou em contexto natural da criança, esses elementos motivacionais não estavam presentes, o que resultou num desempenho inferior no nível de habilidade. Isto indica a necessidade de se avaliar as crianças em contextos ecológicos e com atividades significativas para elas, como visto em Blascovi-Assis ${ }^{21}$.

A falta de estímulos, oportunidades de movimentação e aspectos motivacionais podem ser minimizadas com a inclusão do atletismo de forma lúdica na escola, pois esta atividade trabalha de forma integrada as diferentes habilidades básicas do ser humano.

Os dados mostram também que a ação dos membros superiores é muito importante para melhores resultados da corrida, o que também é evidenciado quando a criança espontaneamente utiliza este segmento corporal de forma mais eficaz em uma situação de prova de atletismo, como ocorreu no festival. A inclusão do atletismo na escola pode levar a situações em que a criança seja levada a experimentar diferentes maneiras de utilizar este segmento para atingir maiores distâncias em menores tempos e assim aperfeiçoar esta habilidade, que será importante em outras situações de sua vida, que não apenas a de prática de esportes.

Desta forma caberia aos profissionais da Educação Física proporcionar diferentes estímulos às crianças, através de festivais, jogos lúdicos e brincadeiras, utilizando-se de elementos do atletismo, já que houve diferença significativa entre o gesto motor durante a situação de teste e a situação de festival.

\section{Conclusão}

Embora a maioria das crianças não tenha demonstrado o nível de desenvolvimento motor esperado para a idade, o desempenho da habilidade foi significativamente melhor no contexto de festival de atletismo do que no contexto de teste. O déficit no desenvolvimento motor pode ter sido gerado pela falta de estímulos proporcionados na fase suscetível das habilidades motoras básicas.

Sugere-se que os professores de Educação Física ofereçam oportunidades, como o atletismo, para o desenvolvimento das habilidades motoras de forma lúdica e descontraída, abrangendo os fatores motivacionais para que a criança desenvolva e apresente um melhor desempenho em habilidades básicas, tais como o correr. Como correr é uma habilidade que é utilizada em muitos esportes e em outros contextos do cotidiano é necessário trabalhar para que as crianças consigam atingir o potencial mais elevado de desempenho da corrida ficando mais preparadas para encarar as demandas ambientais futuras.

Propõe-se que a avaliação do nível de desenvolvimento de habilidades motoras seja feita de maneira ecológica, através de festivais, jogos e brincadeiras, fazendo com que esta situação se aproxime do contexto em que a criança está inserida, já queestá pesquisa identificou que na situação de festival o nível de habilidade motora foi positivamente influenciado pelo ambiente em que o cerca.

\section{Agradecimentos}

Agradecemos o apoio financeiro recebido da CAPES e da Universidade onde o estudo foi realizado e a participação de todas as crianças e da escola onde o estudo foi realizado.

\section{Referências}

1. Gomes CLJ. O atletismo como conteúdo pedagógico e formativo. Londrina: PDE; 2008.

2. Matthiesen SQ, Silva MFG, Silva ACL. Atletismo na escola. Motriz rev. educ. fís. 2008;14(1): 96-104.

3. Matthiesen SQ. Atletismo se aprende na escola. Jundiaí: Fontoura; 2009. 
4. Valentini NC. Validity and reliability of the TGMD-2 for Brazilian children. $J$ MotBehav 2012; 44(4): 275-280.

5. Wickstron RL. Fundamental motor patterns. Philadelphia: Lea Febiger; 1983.

6. Meinel K, Schnabel G. Motricidade I: Teoria da Motricidade Esportiva Sob o Aspecto Pedagógico. Rio de Janeiro: Livro Técnico; 1984.

7. Roberton MA, Halverson LE. Developing children: their chaging movement. Philadelphia: Lea Tebiger; 1984.

8. Mc Clenaghan BA, Gallahue DL. Movimientosfundamentales: sudesarrollo y rehabilitación. Buenos Aires: Panamericana; 1985.

9. Ulrich DA. Test of Gross Development: examiner's manual. Austin: PROED; 2000

10. Silva RCR, Malina RM. Nível de atividade física em adolescentes do Município de Niterói. CadSaude Publica 2000; 16(4): 1091-97.

11. Valentini NC. Percepções de competência e desenvolvimento motor de meninos e meninas: um estudo transversal. Movimento 2002; 8(2): 51-62.

12. Bigotti S, Tolocka RE. Desenvolvimento motor, gênero e medidas antropométricas em crianças na infância avançada. Temas Desenvolv 2005; 14: 49-56.

13. Marques I, Catenassi FZ. Restrições da tarefa e padrões fundamentais de movimento: uma comparação entre o chutar e o arremessar. Rev. educ. fis 2005; 16(2): 155-162

14. Katić R., Retelj E, Milat S, Ivanisević S, Gudelj I. Development of motor and specific motor abilities for athletics in elementary school male and female first-graders. Coll Antropol 2008; 32(4): 1141-1147.

15. Pansera SM, De Paula PR, Valentini NC. Educação Física no ensino infantil: sua influência no desempenho das habilidades motoras fundamentais. Cinergis 2008; 9(2): 24-32.
16. Brauner LM, Valentini NC. Análise do desempenho motor de crianças participantes de um programa de atividades físicas. Rev. educ. fis 2009 20(2): 205-216.

17. Silva SB, Vilela-Junior GB, Tolocka RE. Arremessar por cima do ombro e a distancia percorrida pelo implemento. Rev. bras. ciênc. esporte 2009; 23(4): 309-318.

18. Krebs RJ, Duarte MG, Nobre GC, Nazario PF, Santos JOL. Relação entre escores de desempenho motor e aptidão física em crianças com idades entre 07 e 08 anos. Rev. bras. cineantropom. desempenho hum 2011 13(2): 94-98.

19. Gallahue DL, Ozmun JC. Compreendendo o desenvolvimento motor: em bebes, crianças, adolescentes e adultos. São Paulo: Phorte; 2005.

20. Lorson KM, Goodway JD. Gender differences in throwing form of children ages 6-8 years during a throwing game. Res. q. exerc. sport 2008; 79(2):174-182.

21. Blascovl-Assis SM. Lazer para Deficiência Mental: um relato de experiências. In Marcellino NC. (org.) Lúdico, Educação e Educação Física. ljuí: Unijuí; 2013. p 101-112.

22. Schmidt $R$, Wrisberg $C$. Aprendizagem e performance motora: uma abordagem da aprendizagem baseada no problema. Porto Alegre: Artmed; 2001

23. Rose Junior D. Esporte e atividade física na infância e na adolescência. Porto Alegre: Artmed; 2009.

24. Caillos R. Os jogos e os homens: a máscara e a vertigem. Lisboa: Cotovia 1990.

25. Camargo LODL. Educação para o Lazer. São Paulo: Moderna; 1998. 